\title{
3D Align overlay verification using glass wafers
}

\author{
Eric M.J. Smeets ${ }^{* a}$, Frans G.C. Bijnen ${ }^{\mathrm{a}}$, John Slabbekoorn ${ }^{\mathrm{b}}$ and Henk W. van Zeijl ${ }^{\mathrm{b}}$ \\ ${ }^{a} A S M L$ Special Applications. De Run 6501, 5504 DR Veldhoven, The Netherlands \\ ${ }^{\mathrm{b}}$ Delft Institute of Micro Electronics and Submicron technology . Feldmannweg 17, 2628 CT Delft, \\ The Netherlands
}

\begin{abstract}
In the MEMS world, increasing attention is being given to 3D devices requiring dual-sided processing. This requires lithography tools that are able to align a wafer to both its back side as front side. Overlay describes how well front and back side layers are positioned with respect to each other. Currently there is no simple and fast method to qualify the overlay. This paper covers a method of measuring the overlay between front- and back side patterns using a glass substrate. We describe the methods used, special process requirements and measurement data.

The main advantages of the presented method are the simplicity of the concept and the need for only basic fab processing equipment. The substrate employed is re-usable and low cost. The results are as follows:

1. Glass wafers can be used to measure front to back side overlay. The accuracy of the proposed method is better than $100 \mathrm{~nm}(3 \sigma)$ on ASML PAS 5000/5200 machines. On ASML PAS 5500 steppers, the expected accuracy is better than $80 \mathrm{~nm}(3 \sigma)$.

2. The proposed method of measuring the absolute glass shift, from a glass-on-silicon stack, yields unreliable information. This is due to deformation of the glass. An alternative method is described which builds on result 1 (above).

3. Processing of glass wafers has been established, and a glass overlay measurement wafer has been defined.

4. The benefit of Anti Reflective (AR) coatings is suspected, but not yet proven. Minimizing bi-refringency does not play a role in the measurement accuracy of glass wafers for overlay measurements
\end{abstract}

Keywords: MEMS, MOEMS, FTBA, Overlay, Lithography, 3DAlign ${ }^{\mathrm{TM}}$, glass wafer

\section{INTRODUCTION}

Dual sided processing is beneficial for many applications in the semiconductor industry. For certain Micro-ElectroMechanical Systems (MEMS) devices, dual sided processing is actually vital to product creation. In many devices such as a pressure sensor, the performance and quality of operation of the device depends on the positioning of the processed layers on front and back side. An example of this can be seen in Figure 1 where the operation of a pressure sensor is dependent on the Front To Back side Alignment (FTBA) overlay performance. In the creation process of such devices, the silicon wafer is exposed and etched, flipped and re-exposed.

*eric.smeets@asml.com 


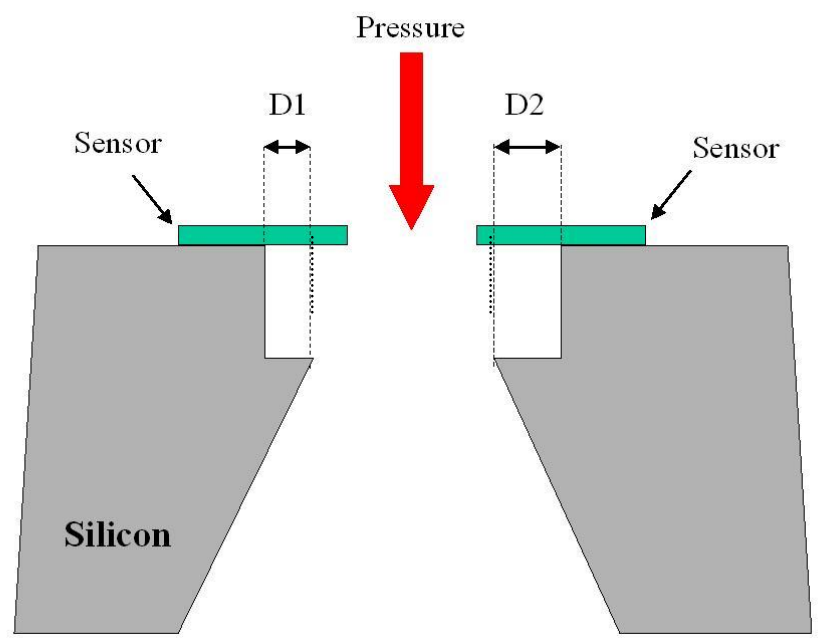

Figure 1: MEMS Pressure sensor. Parameters D1and D2 determine the sensitivity of the pressure detection. This parameter is directly linked to the FTBA overlay (and etch) performance.

It is advantageous to align to the same reference when exposing on either side, in which case, the quality of the exposing tool determines the overlay between the front side and back side of the wafer. In standard ASML lithographic stepper tools, a single Through The Lens (TTL) alignment system is used, employing a red laser beam $(\lambda=632.8 \mathrm{~nm})[6]$. The position determination results from detection of the first orders of the diffracted light from a set of special alignment marks illuminated by the alignment laser.

\section{FRONT TO BACK ALIGNMENT}

Alignment of the front side of a wafer to the back side using a single set of alignment marks is realized using the alignment system as described above, extended with an array of optical elements in the wafer table. Figure 2 shows the optical path in ASML's 3DAlign ${ }^{\mathrm{TM}}$ system [3]. The array consists of lenses and mirrors in a 4F optical arrangement. A mark on the back side of a wafer is illuminated by the laser passing the optical array. The image of the mark is projected into the focal plane of the exposure lens, where it can be picked up by the standard TTL alignment system.
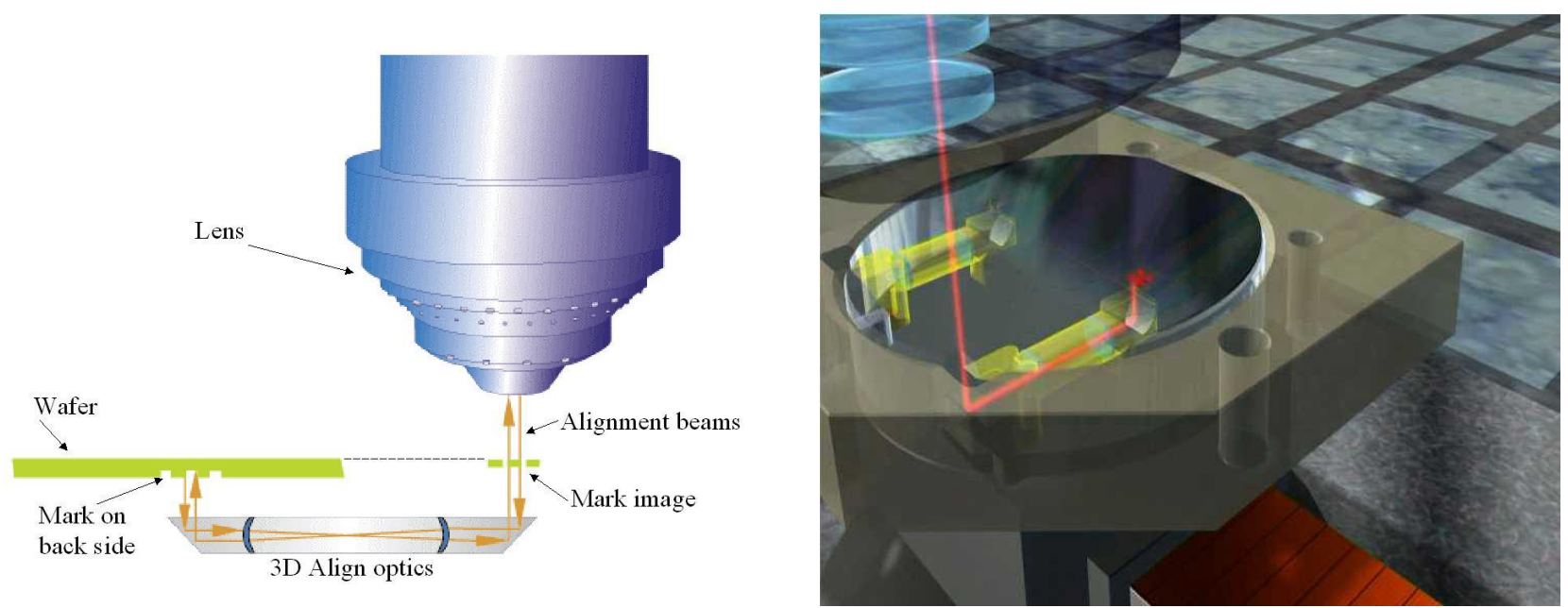

Figure 2 Optical path of 3D Align schematically 
By applying back side alignment metrology [4], the position of a mark on the back side can be determined. Note that the same alignment detection system is used for measuring front side marks. A set of alignment marks (colored dots in Figure 3) can be read from the front side and from the back side. Because the wafer is made out of silicon, it is not possible to have a simultaneous front- and back side readout using alignment laser light at $\lambda=632.8 \mathrm{~nm}$.

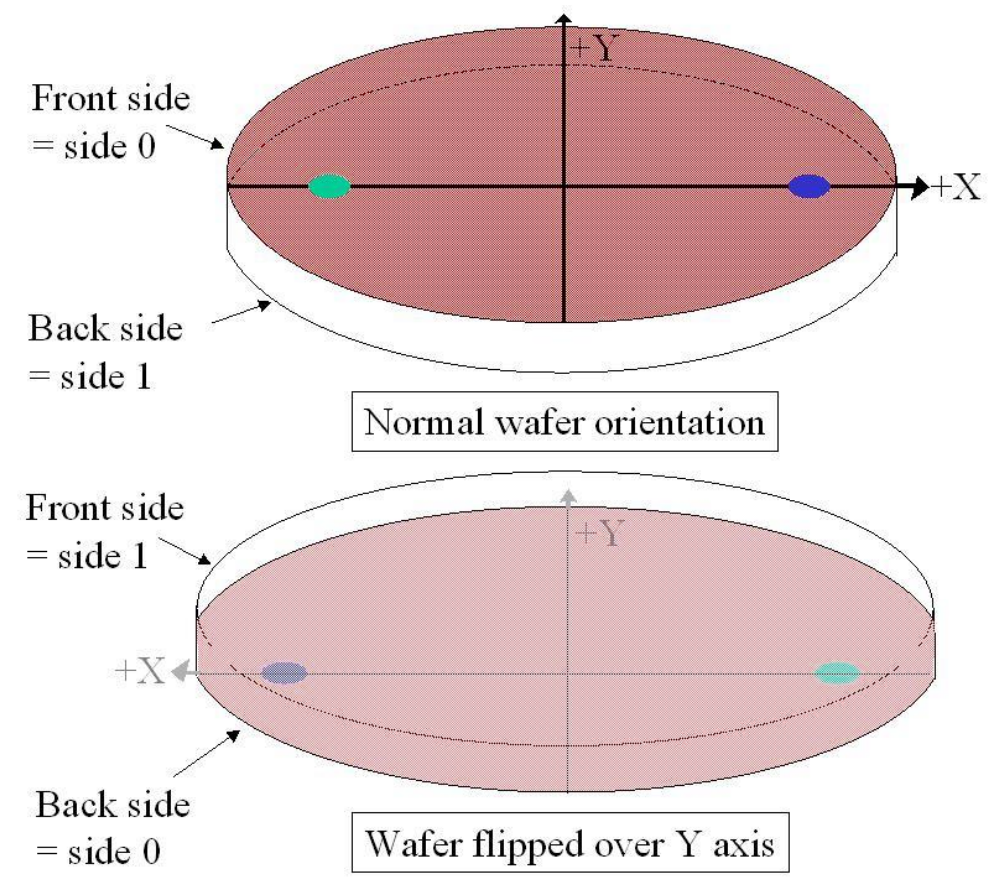

Figure 3 Alignment marks viewed from the top side in two wafer orientations

Given the system as described above, device manufacturing requires knowledge of the FTBA overlay error. This FTBA overlay is defined in Figure 4. This article focuses on the contribution of glass in the FTBA overlay measurement error. 


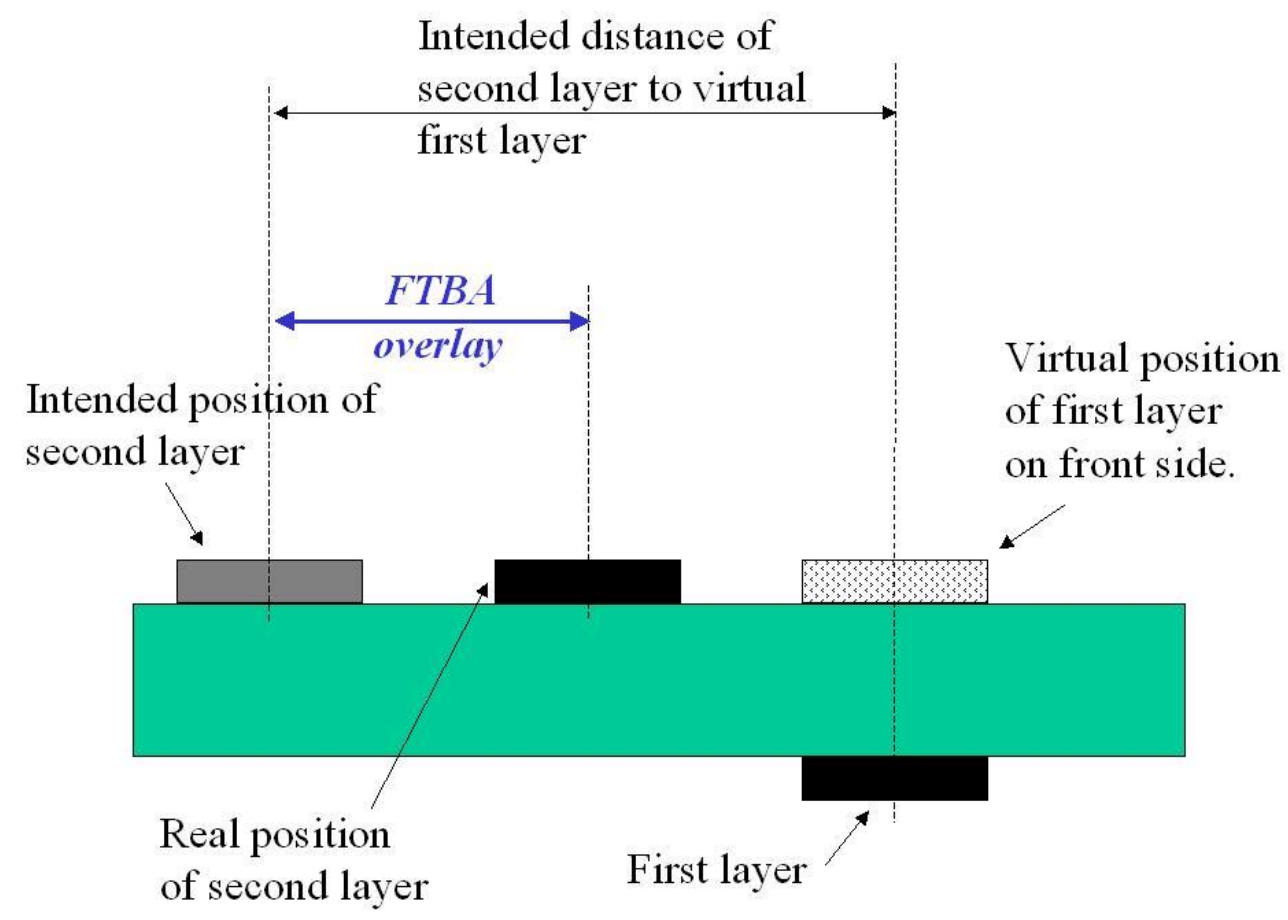

Figure 4 Definition of Front To Back Alignment (FTBA) overlay

\section{STATE OF THE ART}

Measurement of the FTBA overlay is a new technology. Obviously, this is only required in lithographic equipment where alignment on both sides of the substrate (also called Dual Sided Alignment, DSA) is performed. Although present in wafer bonding equipment, definition of an FTBA overlay and its measurement is new in the lithographic field [4]. Measuring the overlay can be accomplished in many ways. A common method is to use infrared alignment to detect back side marks through silicon. Alternatively, non-standard devices may be created on silicon where the device's electrical characteristics depend linearly on the position of the real estate on front and back side. In previous work (see [1], [5]) such a device (resistor array) was used to measure front to back side overlay. The method used there has a measurement accuracy of around $80 \mathrm{~nm}(3 \sigma)$. However, there is need for a method that works in existing lithographic equipment, using existing hardware and existing procedures as much as possible without having to resort to non-standard processing to obtain a measurement. In that respect, the known methods, as described, do not serve this need.

\section{MEASUREMENTS}

Overlay measurement is performed by measuring the first and second layer positions in a similar way to overlay measurement on standard silicon wafers (see Figure 5). The arrows depict the incoming and reflected alignment beam. This beam hits the silicon surface almost perpendicularly. The reflected beam is analyzed, and this leads to the measurement of the position of the mark. 


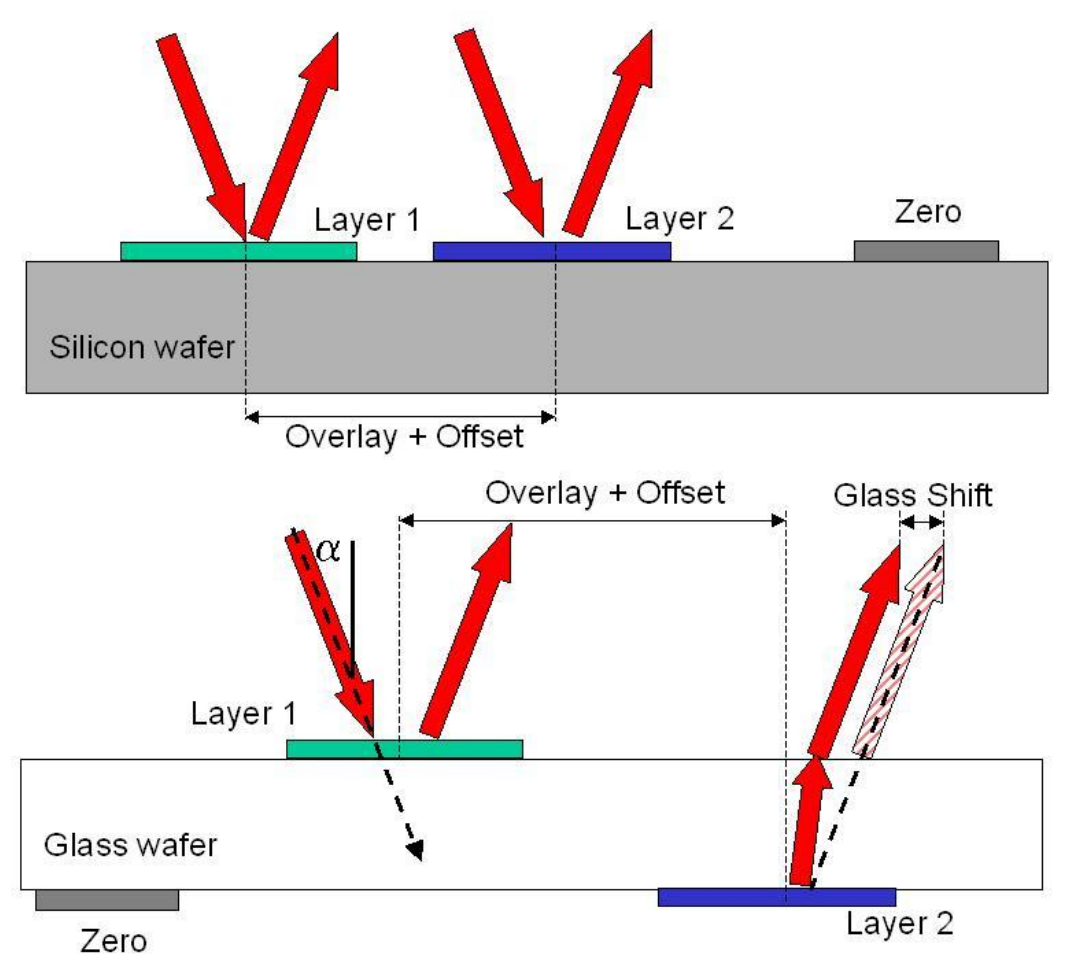

\section{Figure 5 Silicon wafer front side overlay (top) and glass wafer front to back side overlay (bottom). Required height shift corrections are not shown in this Figure.}

Is glass suitable for FTBA overlay measurements?

If glass is fit to act as a substrate for an FTBA verification tool, we need the glass to act homogeneously and show the same behavior throughout the wafer. In short, additional noise due to measuring through glass must be low. For the experiment, we used BK7 and Corning 1737 glass of 500 and $700 \mu \mathrm{m}$ with an overall total thickness variation of less than $2 \mu \mathrm{m}$.

Layer 1 and layer 2 are exposed on the same side of the glass and measured with marks facing down. This closely resembles a standard overlay measurement procedure. The difference is that both layers are not on the front side but at the back, and the orientation of the mark design is mirrored to allow alignment through the glass. In this way the TTL alignment system measures both marks at the bottom with the same orientation. The focus plane remains at the top side of the wafer. Table 1 shows the results of overlay measurements through the glass, compared to silicon. It is anticipated that a single design mark is needed that can be read from the top and through the glass from the back (a so-called omni mark). We have verified that using these omni marks yields no extra measurement offset or noise to an overlay measurement. A further description on omni marks and their use can be found in [2]. The benefit of omni marks is that one can use a single overlay reticle with only one type of mark

Table $1^{*} .3 \sigma$ overlay values using various materials and marks.

\begin{tabular}{|c|c|c|c|c|}
\hline Substrate & Layer 1 & Layer 2 & $\mathbf{X}(3 \sigma)[\mathrm{nm}]$ & $\mathbf{Y}(3 \sigma)[\mathrm{nm}]$ \\
\hline Silicon & Top, PM & Top, PM & 33 & 56 \\
\hline Glass & Bottom, PM & Bottom, PM & 56 & 76 \\
\hline Silicon & Top, PM & Top, Omni & 63 & 68 \\
\hline
\end{tabular}

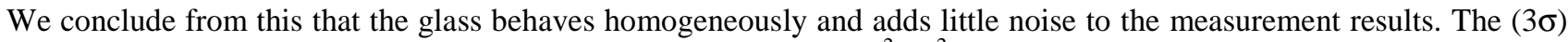
standard deviation between silicon and glass is increased by $\operatorname{sqrt}\left(56^{2}-33^{2}\right)=45 \mathrm{~nm}$ in X and $51 \mathrm{~nm}$ in Y. The use of omni

* In Table 1, no average overlay values are given since this is equipment specific and not of interest here 
marks results in a $3 \sigma$ standard deviation contribution of $54 \mathrm{~nm}$ in $\mathrm{X}$ and $39 \mathrm{~nm}$ in $\mathrm{Y}$. Thus we conclude that omni marks can be used in overlay experiments (with an impact of approximately $50 \mathrm{~nm}$ ).

Can glass be used to measure the incident alignment beam angle?

Measured overlay data needs to be corrected for the influence of the glass on the mark position (see figure 5). If the absolute mark shift introduced by the glass is known, then the FTBA overlay can be calculated as

$$
\text { FTBA overlay }=\text { measured FTBA overlay }- \text { glass shift }- \text { height correction }
$$

The height correction represents the shift in measurement output due to the fact that the layer on the back side is at a position of $\mathrm{z}=-\mathrm{D}$, with $\mathrm{D}$ being the thickness of the glass plate. This factor equals $\mathrm{D}^{*} \tan (\alpha)$ where $\alpha$ is the incident alignment beam angle.

The glass shift represents a shift in measurement output due to the different refractive index of glass with respect to air. The glass influences amount to:

$$
\text { Glass Shift }=\mathrm{D} *(\tan (\alpha)-\tan (\beta))
$$

Where:

$$
\begin{aligned}
& D=\text { thickness of the glass } \\
& \alpha=\text { angle between vertical and the incident alignment beam } \\
& \beta=\text { angle between vertical and the refracted alignment beam in glass }
\end{aligned}
$$

The relationship between $\alpha$ and $\beta$ is given by Snell's law:

$$
\beta=\operatorname{asin}\left(\left(\mathrm{n}_{\mathrm{a}} / \mathrm{n}_{\mathrm{g}}\right) * \sin (\alpha)\right)
$$

Where:

$$
\begin{aligned}
& \mathrm{n}_{\mathrm{a}}=\text { refractive index of air }\left(\text { e.g. } \mathrm{n}_{\mathrm{a}}=1.00027 @ 1000 \text { mbar, } 22^{\circ} \mathrm{C}, 40 \% \mathrm{RH}\right) \\
& \mathrm{n}_{\mathrm{g}}=\text { refractive index of glass }\left(\text { e.g. } \mathrm{n}_{\mathrm{g}}=1.5165 \text { for Corning } 1737 \text { glass }\right) \\
& \alpha=\text { angle between vertical and the incident alignment beam }
\end{aligned}
$$

So, as an example, it follows that when $\alpha=1 \mathrm{mrad}$ and $\mathrm{D}=500 \mu \mathrm{m}$, we find a glass shift of $170 \mathrm{~nm}$ using the refractive index of air and glass as shown above. The fact that the process takes place in a lithographic reduction system is convenient, as environmental conditions are close to constant, keeping $\mathrm{n}_{\mathrm{a}}$ constant as well.

Thus, if we can measure $\alpha$ or $\beta$, then we can calculate the glass shift, calculate the height correction value and thus find the true FTBA overlay.

The glass shift can be found experimentally by measuring a mark position with and without glass. Since the refractive index of air and glass and the glass thickness are known, equations 2 and 3 can be used to calculate $\alpha$. This differential experiment is diagramed in Figure 6. 

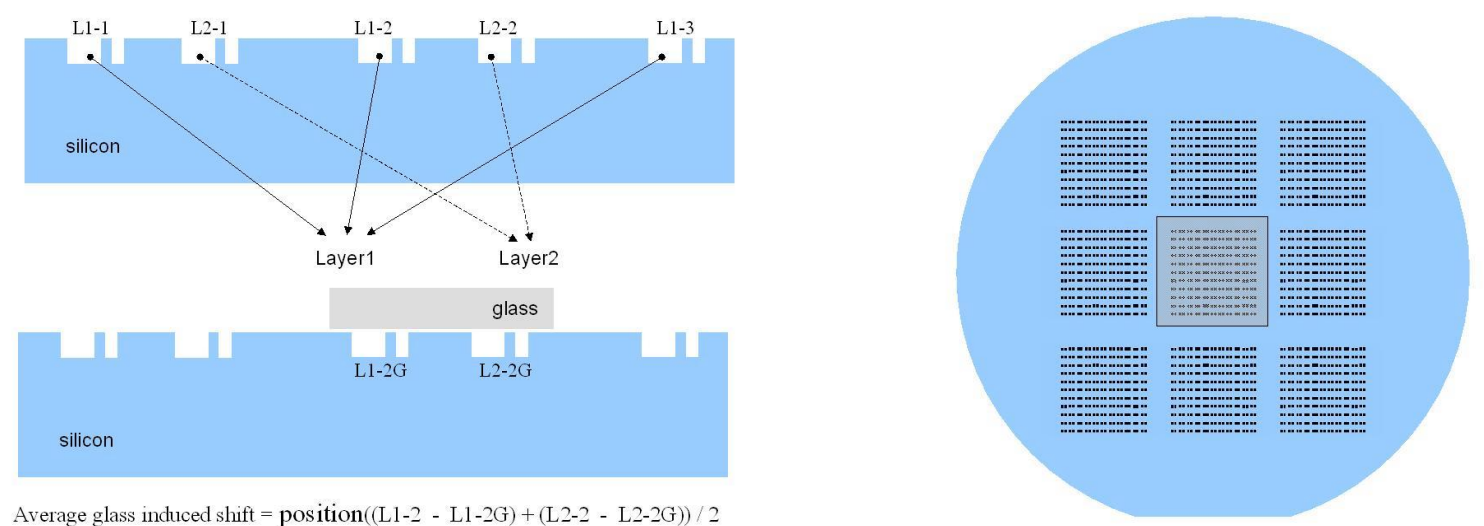

Figure 6 Experiment to determine exact glass shift (left) and wafer with glass (right)

Measuring the position of the marks, and comparing those data with mark positions after some marks have been covered by glass, yields a differential result that reveals the influence of the glass. The measurements are influenced by mark measurement noise with a magnitude of $20 \mathrm{~nm}(3 \sigma)$. Ideally the outcome should reveal a set of vectors equal in length and orientation. The results are shown in Figure 7.

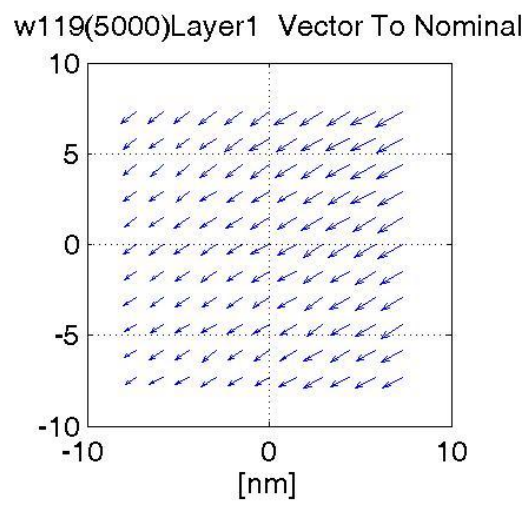

w119(5000)Layer1-X $(\mu, 3 \sigma)=(-213,105)$

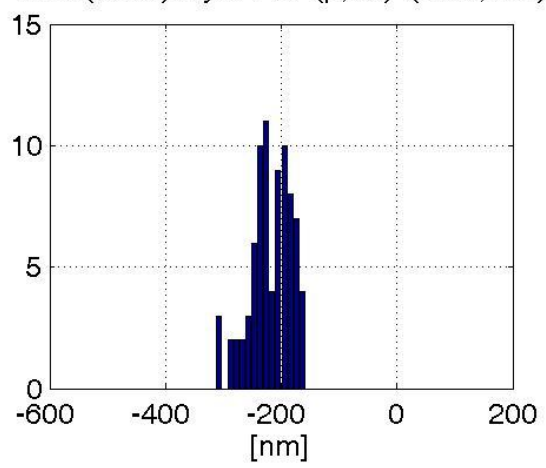

w119(5000)Layer1 Residual error $(\mu=0)$

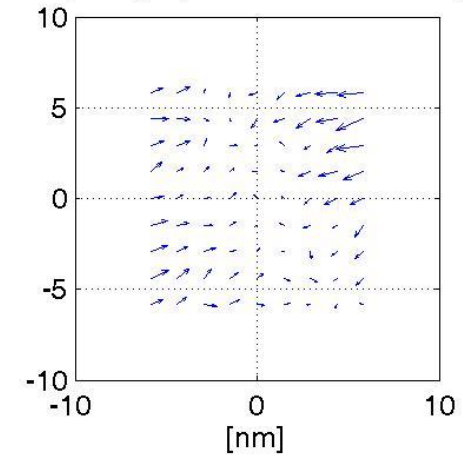

w119(5000)Layer1- $Y(\mu, 3 \sigma)=(-134,47)$

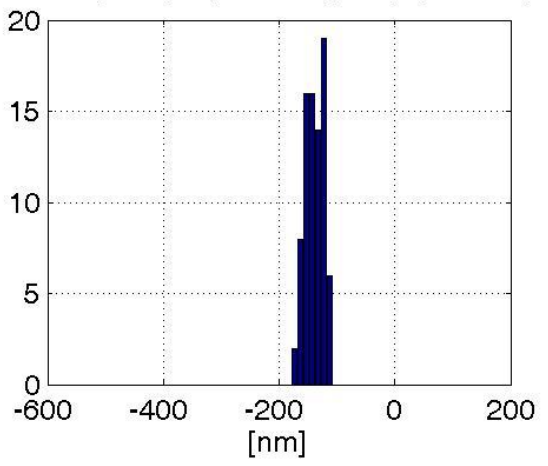

Figure 7 Experimental results of glass on silicon. Glass shift vectors (top left) are not uniform. Glass shift without mean (top right) shows no random error but spherical deformation. The two glass shift distributions for the $X$ direction (left) and $\mathrm{Y}$ direction (right) show a recognizable standard deviation $(3 \sigma)$. 
This indicates that the $3 \sigma$ standard deviation contribution of the glass is in correspondence with the results obtained in the previous section. Investigations show that the adhesion of the glass to the wafer in these experiments causes the glass to deform and introduces stress into the glass. This can be observed from the graph in the upper right corner of figure 7 where clearly a spherical deformation is the main cause for the increase of the standard deviation. Multiple experiments, however, show that the average shift can vary up to $200 \mathrm{~nm}$, using the same glass. This variation in the average shift is so large that the absolute beam angle $\alpha$ cannot be measured accurately enough to be meaningful in the proposed way.

Based on these results, an alternative method has been developed to measure the glass shift. See section 8 , Outlook, for a discussion on this.

Can glass wafers be created for overlay measurements in a standard fab environment?

It is desirable if fabs can develop their own glass wafers. This restricts the type of processes that can be used to create an FTBA overlay verification wafer. Processing experiments show that glass wafers can be created in a fab when the following utilities are present

1) Deposition of $\mathrm{SiO}_{2}$

2) Deposition of aluminum (or other material reflective for alignment laser light)

Standard cleaning agents and baths can be used. However, HF cannot be used, as it affects the glass.

A typical layer thickness to be applied for aluminum is $200-400 \mathrm{~nm}$. A minimum of $200 \mathrm{~nm}$ is required to form a sufficiently opaque reflective layer (to reflect the alignment beam, $\lambda=632.8 \mathrm{~nm}$ ). Minimum requirements for $\mathrm{SiO}_{2}$ depend on the layer it is used in. The bottom layer should be at least $400 \mathrm{~nm}$ thick, as marks are etched into this layer. Mark depth typically is around $120 \mathrm{~nm}$. $\mathrm{SiO}_{2}$ layer thickness should not exceed $600 \mathrm{~nm}$ to avoid undesired stress in the glass. Lab results indicate that the processing is not more critical than in common silicon processing. Most fabs will therefore be able to create glass wafers for overlay measurements themselves.

What is the effect of Anti Reflective (AR) layers and bi-refringency on glass wafer measurement accuracy?

Some alignment systems are sensitive to reflections. An example is the standard phase modulated TTL alignment systems in PAS 5000/5200 machines. Measurements of the glass shift using glass-on-silicon stacks have been performed using untreated glass and AR coated glass. The AR coating is formed by two layers: the first one on the glass is $\mathrm{Ta}_{2} \mathrm{O}_{5}$ or $\mathrm{TiO}_{2}$, the second one $\mathrm{SiO}_{2}$. Untreated glass $\mathrm{BK} 7$ (bi-refringency value: $10(\mathrm{~nm} / \mathrm{cm}) /\left(\mathrm{kg} / \mathrm{mm}^{2}\right)$ ) and Corning 1737 (birefringency value: $\left.333(\mathrm{~nm} / \mathrm{cm}) /\left(\mathrm{kg} / \mathrm{mm}^{2}\right)\right)$ were used. Especially, the influence of bi-refringency is difficult to predict, and experimental results on this matter are relevant.

Table 2. Glass shift standard deviation $(3 \sigma)$ using ARC/non ARC glass plates.

\begin{tabular}{|l|l|l|l|}
\hline Substrate & ARC/No ARC & $\begin{array}{l}\text { Glass shift } \\
\mathbf{X}(\mathbf{3} \sigma)[\mathbf{n m}]\end{array}$ & $\begin{array}{l}\text { Glass shift } \\
\mathbf{Y ~}(\mathbf{3} \sigma)[\mathbf{n m}]\end{array}$ \\
\hline 1 & ARC & 41 & 57 \\
\hline 2 & ARC & 57 & 72 \\
\hline 3 & ARC & 104 & 238 \\
\hline 4 & ARC & 29 & 37 \\
\hline 5 & No ARC & 166 & 315 \\
\hline 6 & No ARC & 171 & 220 \\
\hline
\end{tabular}

Table 3. Glass shifts standard deviation $(3 \sigma)$ using BK7 and Corning 1737 glass

\begin{tabular}{|c|c|c|c|}
\hline Substrate & Туре & $\begin{array}{l}\text { Glass shift } \\
X(3 \sigma)[\mathrm{nm}] \\
\end{array}$ & $\begin{array}{l}\text { Glass shift } \\
\text { Y }(3 \sigma)[\mathrm{nm}]\end{array}$ \\
\hline 1 & BK7 & 166 & 356 \\
\hline 2 & BK7 & 390 & 286 \\
\hline 3 & BK7 & 188 & 305 \\
\hline 4 & Corning 1737 & 166 & 315 \\
\hline 5 & Corning 1737 & 171 & 220 \\
\hline
\end{tabular}


The benefit of an AR coating seems to be indicated by the data in Table 2. As these data were found with the glass plate to silicon attachment sequence as discussed before, we remain skeptical regarding the benefits of AR coating. Theoretically, the better results in case of AR layers could be generated by "lucky" glass plate attachments. Birefringency (Table 3) does not play a role in the accuracy of glass wafer overlay measurement, as it does not influence the standard deviation positively or negatively.

\section{GLASS WAFER LAYOUT}

Based on the experimental data, we can construct a glass wafer to be used in overlay experiments. Material properties of glass closely resemble the material properties of silicon. The most striking differences are the heat conductivity index (which for glass is around $80-100$ times lower than for silicon) and the presence of sodium (Na) in many varieties of glass. As the metal $\mathrm{Na}$ is unwanted in a standard silicon wet treatment environment, we have to make provisions to keep the sodium in the glass. A way to provide this is to cover the glass with a protective layer. Also, some silicon dioxide for protection of the back side layer is advised. Thus, the following stack on glass (see Figure 8) is suited for FTBA overlay measurements.

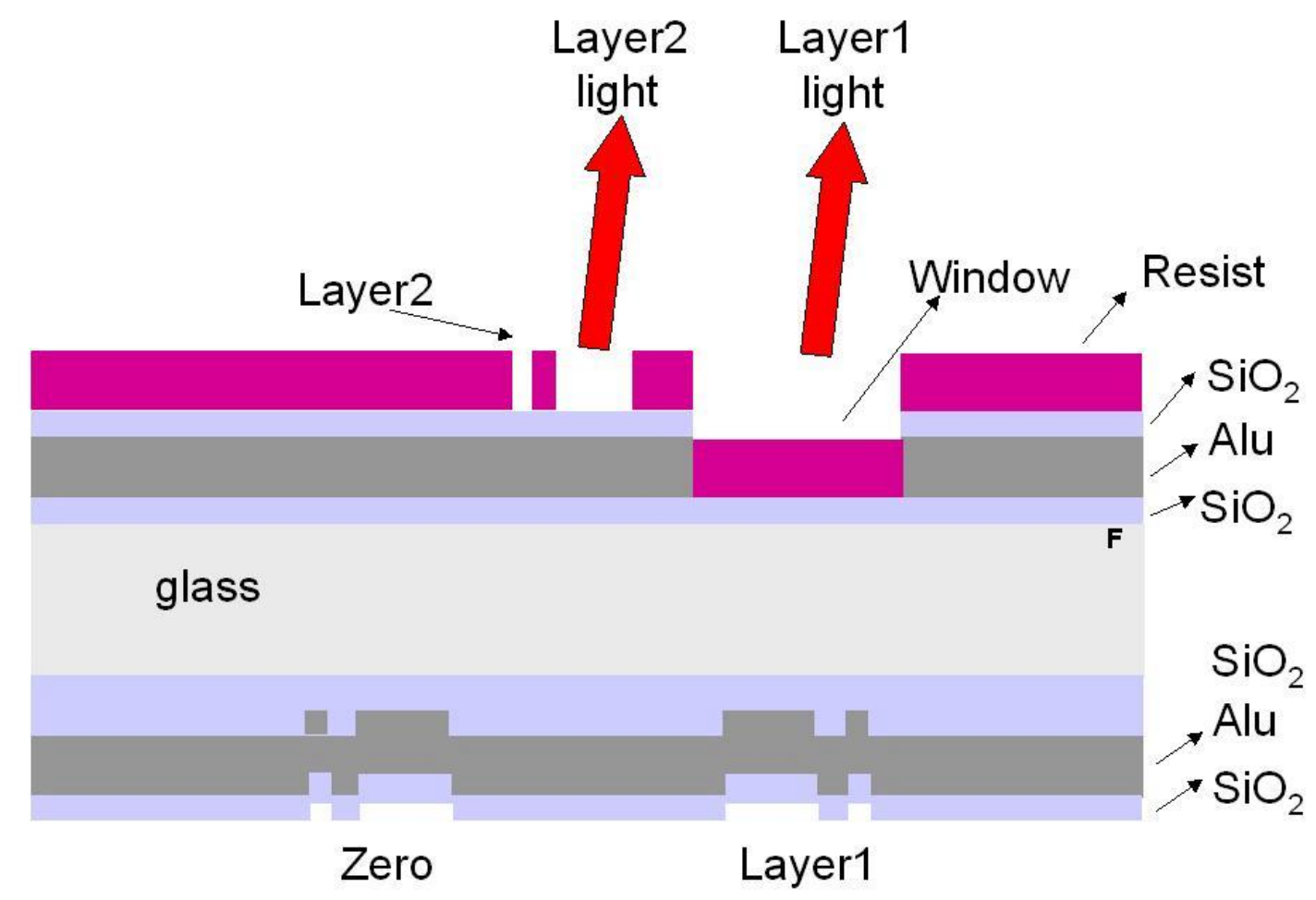

Figure 8 Glass stack for FTBA overlay measurements

The $\mathrm{SiO}_{2}$ layer at the very bottom is for aluminum protection only. The $\mathrm{SiO}_{2}$ layer between layer 1 and the glass hosts the marks. The aluminum layer at the top provides a reflective layer for the alignment beam. This layer is necessary because proper reflection is required to enable alignment on the front side mark. The top $\mathrm{SiO}_{2}$ layer protects the aluminum from being affected by the developer. With this layout, the glass wafer can be used multiple times by just removing the resist, spinning a fresh layer and exposing layer 2 again. As layer 1 is machine specific, we can also use this glass wafer for machine-to-machine matching (A to B matching). 


\section{DISCUSSION}

The question of accuracy of the measurement method with glass has actually not been answered. We may deduce, however, from the second experiment that the noise the glass adds to the FTBA overlay measurement is within the expected accuracy. Differential measurements with and without glass on silicon did not yield reliable results, as the process of attaching the glass to the silicon is tedious, difficult and error-prone. This step however was only required to determine the beam angle required for the calculation of the FTBA overlay value. The accuracy of the beam angle can be added to the currently found values for the FTBA overlay accuracy contributors. There are more methods to determine the beam angle in an accurate way. One of these, which does not suffer from the quality of glass-to-silicon attachment, is proposed in the outlook.

\section{CONCLUSIONS}

The question was posed if, and how well, glass wafers can be used to measure 3D Align overlay. Experiments show that measuring both overlay layers through glass can be done with a $(3 \sigma)$ standard deviation within $10-20 \%$ of the standard deviation of normal front side alignment on silicon. This means that the glass used behaves homogeneously. For ASML PAS5000/5200 systems, we can estimate a 3D overlay measurement accuracy less than $100 \mathrm{~nm}$. For ASML PAS 5500 steppers, an accuracy better than around $80 \mathrm{~nm}$ is expected.

The conducted experiments have not lead to a reliable method to measure the absolute glass shift but have increased understanding on glass wafer processing. The proposed glass stack has been realized and can be used to measure 3D Align overlay.

\section{OUTLOOK}

It is possible to calculate the exact influence of the glass shift by exposing marks on both sides of a glass wafer and measuring the position of the marks twice. In the first measurement the wafer is in its normal position, and in the second, the wafer has been rotated by $180^{\circ}$ in the horizontal plane. Figure 9 shows this graphically.

The advantage of this method is that the glass shift, used to compensate the measurement, can be calculated in a straightforward way. This method can be used to determine the alignment beam angle $\alpha$ independent of the quality of the silicon to glass attachment. 

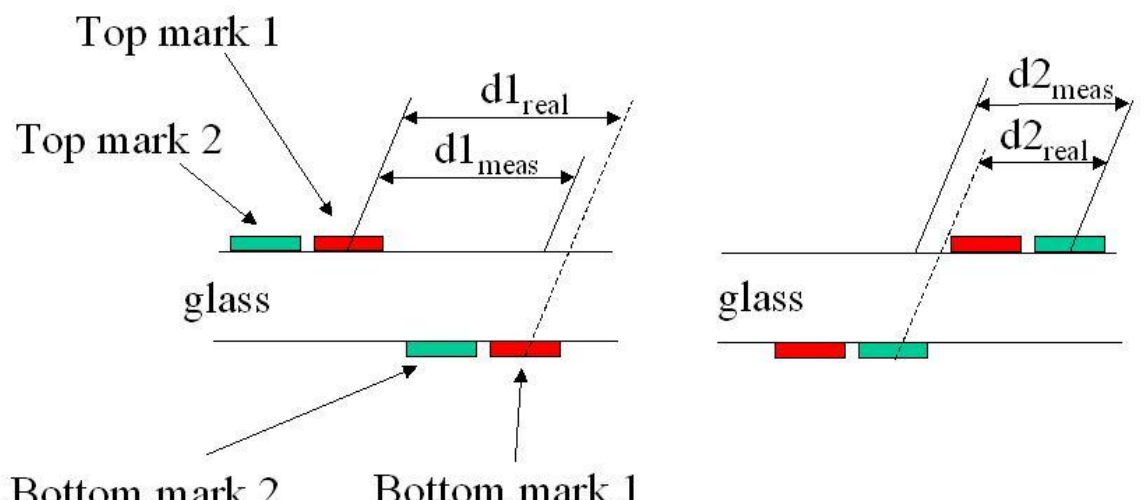

\section{Bottom mark 2 Bottom mark 1}

$$
\text { Orientation } 0^{\circ}
$$

Orientation $180^{\circ}$

$$
\text { Glass shift }=F\left(d 2_{\text {meas }}-d 1_{\text {meas }}\right)
$$

\section{Figure 9 Rotation method to determine glass influences. A top mark and a bottom mark are measured twice: first in a $0^{\circ}$ oreintation $\left(\mathrm{d}_{\text {meas }}\right)$, then in a $180^{\circ}$ orientation $\left(\mathrm{d} 2_{\text {meas }}\right)$. The glass shift is a function $\mathrm{F}$ of the difference of the two measurements.}

At the time this paper was written, no data on this approach was available yet. We anticipate that this method yields absolute glass shift information and that the method acts robustly. For some ASML lithography systems, special wafer or wafer handling requirements must be in place to enable the $180^{\circ}$ rotation.

\section{REFERENCES}

[1] H.W. van Zeijl and J. Slabbekoorn, "Characterization of Front- to Backwafer Alignment and Bulk Micromachining Using Electrical Overlay Test Structures", by . Journal of MicroMechanics and Micromachining, Issue 4

[2] H.W. van Zeijl and J. Slabbekoorn et al., "Backwafer optical lithography and wafer distortion in substrate transfer technologies", Proc. SPIE Vol. 4148, 2000, pp 200-207

[3] C. Q. Gui, W. v. Buel, F. G. Bijnen and J. Lof, "Extended front-to-back alignment capability for MEMS/MOEMS applications", Proc. SPIE Vol. 4688, 2002, pp 867-873

[4] F. G. Bijnen, W. van Buel, C. Q. Gui and J. Lof, "Front-to-back alignment metrology", Proc. SPIE Vol. 5037, 2003, pp. 641-646

[5] H. W. van Zeijl, F. G. Bijnen and J. Slabbekoorn, "Characterization of waferstepper and process-related front- to backwafer overlay errors in bulk micromachining using electrical overlay test structures”, Proc. SPIE Vol. 5455, 2004, pp 398-406

[6] S. Wittekoek, M. van den Brink, H. Linders, J. Stoeldrayer, J.W.D. Martens and D. Ritchle, "Deep UV wafer stepper with through the lens wafer to reticle alignment", Proc SPIE Vol 1264, 1990, pp 534-546 